\title{
Connaissance paysanne des insectes foreurs de tiges du riz et leurs dégâts dans différentes zones écologiques du Bénin (Afrique de l'Ouest)
}

\author{
Abou Togola ${ }^{1}$ \\ Francis E. Nwilene ${ }^{2}$ \\ Alphonse Agbaka ${ }^{3}$ \\ Florence Anato ${ }^{3}$ \\ Tolulope A. Agunbiade ${ }^{2}$ \\ Daniel C. Chougourou ${ }^{3}$ \\ ${ }^{1}$ Centre du Riz pour l'Afrique (AfricaRice) \\ Division Entomologie \\ 01 BP 2031 \\ Cotonou \\ Bénin \\ $<$ a.togola@cgiar.org> \\ ${ }^{2}$ Centre du Riz pour l'Afrique (AfricaRice) \\ P.M.B. 5320 \\ Oyo Road \\ Ibadan \\ Nigeria \\ $<$ <.nwilene@cgiar.org> \\ $<$ tolulope_adeniran@yahoo.com> \\ 3 École polytechnique d'Abomey Calavi \\ (EPAC) \\ 01 BP 2009 \\ Cotonou \\ Bénin \\ <agbalph@yahoo.fr> \\ <anatoflorence@yahoo.fr> \\ <chougouroud@yahoo.de>
}

\begin{abstract}
Résumé
Au Bénin, les dommages causés par les foreurs de tiges constituent une menace pour la production rizicole. La présente étude vise à évaluer la connaissance paysanne de ces ravageurs en vue de trouver une solution participative à leur surveillance et leur contrôle en milieu rural. Ainsi, une enquête agronomique a été menée auprès de 151 producteurs des départements du Zou, des Collines et du Couffo où des spécimens des foreurs de tige du riz et les échantillons de leurs dégâts ont été présentés aux paysans pour identification. De plus, des visites ont été organisées dans les champs paysans afin de vérifier les connaissances des riziculteurs sur le terrain. Les résultats obtenus montrent que les paysans ont une bonne connaissance des principaux foreurs de tige tels que Sesamia calamistis Hampson (Lepidopera : Noctuidae), Chilo zacconius Bleszynski (Lepidoptera : Pyralidae), Maliarpha separatella Ragonot (Lepidopera: Pyralidae) et Diopsis spp. (Diptera : Diopsidae). Les dégâts des foreurs, notamment les cours morts et les panicules blanches, sont également très bien reconnus par les paysans. La connaissance avérée de ces foreurs par les agriculteurs béninois permettrait aux services d'encadrement rural de mettre à profit l'expérience et le savoir des paysans en les associant aussi bien aux diagnostics locaux qu'aux opérations de lutte contre ces ravageurs.
\end{abstract}

Mots clés : Bénin ; dégât ; diptera ; insecte déprédateur de tige ; lepidoptera.

Thèmes : pathologie ; productions végétales.

\section{Abstract \\ Farmer knowledge of rice stem borers and their damage in various ecological zones of Benin (West Africa)}

In Benin, damage caused by stem borers are a threat to rice production. The present study aimed at evaluating farmer knowledge of these pests in order to find participative solutions for their monitoring and control in rural areas. An agronomic survey was carried out with 151 producers in the regions of Zou, Collines and Couffo where specimens of rice stem borers and samples of their damage were exhibited to farmers for identification. Field visits were also undertaken in order to observe farmer knowledge. The results recorded show that rice producers in Benin have good knowledge of the main rice stem borers such are Sesamia calamistis Hampson (Lepidopera : Noctuidae), Chilo zacconius Bleszynski (Lepidoptera : Pyralidae), Maliarpha separatella Ragonot (Lepidopera: Pyralidae) and Diopsis spp. (Diptera: Diopsidae). Damages caused by stem borers, such as white panicle and dead heart, are also very well recognized by the farmers. The proven knowledge of rice stem borers by farmers would allow agricultural services to take advantage of farmers' experience and knowledge by associating them in local diagnosis and pest control measures.

Key words: Benin; damage; diptera; lepidoptera; stem eating insects.

Subjects: pathology; vegetal productions.

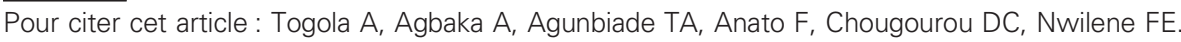
Connaissance paysanne des insectes foreurs de tiges du riz et leurs dégâts dans différentes zones écologiques du Bénin (Afrique de l'Ouest). Cah Agric 2010; 19: 262-6. DOI: 10.1684/agr.2010.0410. 
e riz (Oryza spp.) est devenu l'une des céréales les plus consommées au Bénin. La production locale n'arrive plus à satisfaire les besoins de la population et le pays doit importer chaque année des milliers de tonnes de cette céréale (Assigbe, 2003).

Afin de réduire cette dépendance vis-à-vis de l'extérieur, la production locale doit être encouragée. Cependant, les attaques occasionnées par les insectes en général et les foreurs de tige en particulier limitent de nos jours la production locale du riz. Dans les années de forte infestation, les foreurs seuls sont capables d'engendrer des pertes pouvant atteindre $30 \%$ sur le riz pluvial et $100 \%$ sur le riz de bas-fonds (Umeh et al., 1993). La lutte contre eux doit passer nécessairement par une surveillance régulière de leurs populations et dégâts.

Au Bénin, la surveillance et l'alerte précoce concernant les ravageurs sont dévolues aux seuls services d'encadrement, mais, compte tenu de leurs moyens limités, ils n'arrivent pas à cerner à temps tous les problèmes entomologiques. La prise en compte de la participation paysanne dans les diagnostics locaux est très limitée (Velay et al., 2001), car très peu d'agronomes admettent que les agriculteurs connaissent suffisamment les réalités de leurs terroirs (Friedberg, 1999). Or, le rôle des paysans dans de telles opérations est très important, car ils sont les premiers témoins des problèmes qui apparaissent dans leurs champs. Cependant, avant de solliciter la participation des agriculteurs dans les diagnostics locaux, il parait prioritaire de savoir si oui ou non, ils ont une bonne connaissance des phénomènes entomologiques. L'objectif de cette étude est de mettre en évidence la connaissance des agriculteurs sur les foreurs de tige du riz et leurs dégâts dans les zones écologiques des plateaux, des bas-fonds et zones irriguées du Bénin en vue de justifier leur participation dans les programmes de lutte entomologique. En outre, l'étude permettra d'identifier la présence des principaux foreurs de tige et leur répartition à travers les zones de production du riz afin de mieux orienter les interventions futures pour une meilleure protection des cultures rizicoles au Bénin.

\section{Matériel et méthode}

Au cours de la campagne 2006, le Centre du riz pour l'Afrique (AfricaRice) en collaboration avec l'Institut national des recherches agricoles du Bénin (Inrab) a conduit une enquête, dans les départements du Zou, des Collines et du Couffo où les agriculteurs de huit villages ont été interrogés: Koussin, Lélé, Dévé, Sowé, Gankpètin, Sokponta, Lema, et Odo-Otchèrè. Le choix des sites a été fait en considérant l'importance de la culture du riz dans les localités. Des entretiens individuels ont été menés auprès de 151 producteurs de riz (hommes et femmes) choisis de manière aléatoire à l'intérieur des zones de production du riz, prenant en compte les différents sites (bas-fonds, plateaux et zones irriguées). Les réponses paysannes étaient notées sur les questionnaires. Par ailleurs, les enquêteurs étaient munis des spécimens des principaux insectes foreurs du riz (Sesamia calamistis, Chilo zacconius, Maliarpha Separatella et Eldana saccharina, Diopsis spp. et Orseolia oryzivora), d'échantillons de cours morts et de panicules blanches ainsi que de photographies des principales chenilles foreuses et symptômes de leurs dégâts sur le riz (Chaudhary et al., 2003). La procédure de l'enquête comprenait l'interview des paysans et la visite des champs.

L'interview a consisté à rencontrer chaque producteur individuellement et à lui présenter les spécimens et photos d'insectes foreurs (adultes et larves) et des échantillons de leurs dégâts. Pour chaque insecte présenté, il a été demandé au producteur de donner son nom vernaculaire, de faire sa description, d'indiquer ses dégâts et les stades phénologiques vulnérables et enfin de citer les organes de la plante où on le rencontre. Pour chaque dégât présenté, le producteur devait indiquer sa nature, ses causes, les stades phénologiques de son apparition et son importance économique. Enfin, les pertes de production dues aux foreurs étaient estimées par les producteurs sur la base du nombre de sacs de $100 \mathrm{~kg}$ perdus annuellement. Ainsi, trois niveaux de pertes ont été considérés : perte faible (inférieur à $10 \%$ de la production), moyennement grande (entre $10 \%$ et $20 \%$ ) et élevée (dépassant $20 \%$ de la production).

La visite des champs a pris en compte un seul champ par paysan, celui qui se trouvait aux stades vulnérables du tallage ou de l'épiaison (stades vulnérables aux foreurs). Au champ, le paysan devait trouver et montrer aux enquêteurs les dégâts des foreurs (cours morts, panicules blanches, galles et tunnels). Ensuite, il devait disséquer les tiges infestées, extraire le foreur, donner son nom vernaculaire, le décrire et enfin le montrer dans la collection de référence ou sur la photographie. Pour les foreurs de l'ordre des Lépidoptères l'identification portait seulement sur les larves qui sont facilement observables dans les tiges infestées. Quant à ceux appartenant à l'ordre des Diptères, l'identification concernait les larves et les adultes. Pour un foreur non rencontré sur le terrain, des questions complémentaires étaient posées au paysan pour savoir s'il avait une connaissance réelle de l'espèce concernée. Enfin, chaque producteur a évalué la perte en fonction des dégâts présents au champ. Les réponses paysannes étaient notées sur les questionnaires. Les données collectées ont été réorganisées sous forme de variables quantitatives après codification. Elles ont été ensuite saisies dans le logiciel Excel et soumises à l'analyse statistique à l'aide du logiciel SAS (Statistical Analysis System) version 9.1 (SAS Institute, 2003). Dans le cas de cette étude, les statistiques descriptives ont été utilisées pour faire ressortir les pourcentages moyens des réponses paysannes pour chaque variable étudiée suivant les différentes zones écologiques.

\section{Résultats}

\section{Connaissance des agriculteurs sur les foreurs de tige du riz}

Les résultats des interviews (RI), ont montré que toutes les espèces de foreurs de tige du riz présentées sont connues des paysans (tableau 1). Dans les zones de riziculture de plateau, C. zacconius et $M$. Separatella ont été les plus reconnus (soit par $83 \%$ des producteurs). Ils ont été suivis par les deux Diptères Diopsis spp. et O. oryzivora (par $75 \%$ des paysans) et enfin $S$. calamistis et $E$. saccharina (par $50 \%$ des paysans). Dans les zones écologiques de bas-fond, $M$. Separatella, C. zacconius et Diopsis spp. ont été les plus reconnus par les producteurs. Enfin, dans la zone de riziculture irriguée, S. calamistis, Diopsis spp. et C. zacconius ont été les plus reconnus tandis que M. separatella, E. saccharina et O. oryzivora ont été les moins reconnus par les paysans. Les observations au champ ont permis de vérifier les connaissances paysannes sur le terrain où Diopsis spp. a été correctement identifié par $90 \%$ d'entre eux, C. zacconius par $83 \%, S$. calamistis par $62 \%$ et $M$. Separatella par $47 \%$. En revanche, la présence des espèces 
E. saccharina et O. oryzivora n'a pas été constatée au champ. Toutefois, les producteurs ont su décrire E. saccharina et ont expliqué que cette espèce se rencontre sur le riz quand cette culture se trouve à côté d'un champ de maîs. Les résultats des observations au champ (RC) sont notés dans le tableau 1.

Au vu des résultats des interviews et des observations au champ, il s'avère que les paysans des principales zones de production du riz au Bénin connaissent au moins les quatre foreurs de tige que sont Diopsis spp, C. zacconius, S. calamistis et M. Separatella. Pour ce qui concerne E. saccharina et O. oryzivora, leur connaissance par les paysans n'a pas été pratiquement prouvée.

\section{Connaissance des agriculteurs sur les dégâts des foreurs de tige du riz}

Au cours de l'interview, tous les dégâts des foreurs présentés ont été déclarés connus par les paysans, les cœurs morts et les panicules blanches l'ayant été davantage que les autres. Les observations au champ ont permis à $98 \%$ des producteurs d'identifier correctement les panicules blanches et à $85 \%$ d'entre eux d'identifier les cours morts. Seuls $29 \%$ ont pu reconnaître les tunnels dans les tiges (tableau 1). Pour les dégâts des galles, ils n'ont pas été vus au champ: les paysans les confondaient avec le symptôme de mauvais déploiement des feuilles suite aux attaques tardives des Diopsides.

L'importance économique des dégâts de panicules blanches a été signalée par $59 \%$ des paysans enquêtés alors que celle des cours morts, des tunnels et des galles a été mentionnée respectivement par 21, 13 et $7 \%$ des producteurs (tableau 2 ).

Ces résultats montrent que les producteurs ont une bonne connaissance des principaux dégâts des foreurs de tige du riz au Bénin et qu'ils maîtrisent ceux qui sont économiquement importants.

\section{Connaissance des agriculteurs sur les stades phénologiques vulnérables aux foreurs du riz}

Dans l'ensemble des zones de production, $42 \%$ des paysans interrogés ont estimé que le stade épiaison est le plus sensible aux attaques des foreurs de tige, $37 \%$ ont cité le stade tallage comme étant le plus sensible et $18 \%$ ont trouvé que le stade plantule (prétallage) est le plus vulnérable (tableau 2)
Tableau 1. Proportion des producteurs ayant identifié les foreurs de tige et leurs dégâts dans chaque zone écologique.

Table 1. Proportion of farmers having identified stem borers and their damage in each ecological zone.

\begin{tabular}{|c|c|c|c|c|c|c|c|c|}
\hline & \multicolumn{8}{|c|}{ Pourcentage de paysan (\%) } \\
\hline & \multicolumn{2}{|c|}{ Bas-fonds } & \multicolumn{2}{|c|}{ Plateau } & \multicolumn{2}{|c|}{$\begin{array}{c}\text { Zone } \\
\text { irriguée }\end{array}$} & \multicolumn{2}{|c|}{ MG } \\
\hline & $\mathbf{R I}$ & $\mathbf{R C}$ & $\mathbf{R} \mathbf{I}$ & $\mathrm{RC}$ & $\mathbf{R I}$ & RC & $\mathbf{R I}$ & $\mathbf{R C}$ \\
\hline \multicolumn{9}{|c|}{ 1. Espèces de foreurs de tige } \\
\hline Sesamia calamistis & 41 & 61 & 50 & 80 & 75 & 46 & 55 & 62 \\
\hline Chilo zacconius & 61 & 78 & 83 & 87 & 73 & 84 & 72 & 83 \\
\hline Eldana saccharina & 42 & - & 50 & - & 15 & - & 36 & - \\
\hline Orseolia oryzivora & 52 & - & 75 & - & 41 & - & 56 & - \\
\hline Diopsis sp. & 59 & 93 & 75 & 82 & 74 & 96 & 69 & 90 \\
\hline Maliarpha separatella & 69 & 43 & 83 & 57 & 45 & 42 & 66 & 47 \\
\hline \multicolumn{9}{|c|}{ 2. Dégâts des foreurs de tige } \\
\hline Cœur mort & 80 & 77 & 92 & 86 & 96 & 93 & 89 & 85 \\
\hline Panicule blanche & 89 & 95 & 100 & 100 & 98 & 98 & 96 & 98 \\
\hline Galle & 79 & - & 100 & - & 70 & - & 83 & - \\
\hline Tunnels & 72 & 20 & 92 & 18 & 88 & 49 & 84 & 29 \\
\hline
\end{tabular}

Chaque moyenne correspond au pourcentage de paysans ayant reconnu les foreurs de tige ou le dégât concernés dans la zone écologique indiquée.

$\mathrm{RI}$ : résultats suite aux interviews ; RC : résultats au champ ; - : aucune donnée existante. $M G$ = moyennes générales.

\section{Tableau 2. Connaissance des agriculteurs de l'importance économique des dégâts des foreurs de tige et des stades phénologiques vulnérables du riz.}

Table 2. Farmer knowledge of economic importance of stem borer damage and the different susceptibility of rice according to its stage.

\begin{tabular}{|c|c|c|c|c|}
\hline & \multicolumn{4}{|c|}{ Pourcentage de paysans (\%) } \\
\hline & Bas-fonds & Plateau & Zone irriguée & MG \\
\hline \multicolumn{5}{|c|}{ 1. Les types de dégâts } \\
\hline Cœur mort & 24 & 25 & 13 & 21 \\
\hline Panicule blanche & 52 & 67 & 58 & 59 \\
\hline Galle & 7 & 8 & 6 & 7 \\
\hline Tunnels des tiges & 17 & 0 & 23 & 13 \\
\hline Total $(\%)$ & 100 & 100 & 100 & 100 \\
\hline \multicolumn{5}{|c|}{ 2. Les stades phénologiques } \\
\hline Plantule & 15 & 17 & 21 & 18 \\
\hline Tallage & 28 & 33 & 50 & 37 \\
\hline Épiaison & 47 & 50 & 29 & 42 \\
\hline Total (\%) & 100 & 100 & 100 & 100 \\
\hline
\end{tabular}

NB : les nombres de la section 1 sont les pourcentages de paysans de la zone écologique indiquée qui déclarent que le type de dégât concerné est le plus important sur le plan économique.

Les nombres de la section 2 sont les pourcentages de paysans de la zone écologique indiquée qui déclarent que le stade concerné est le plus vulnérable aux foreurs de tige. MG = moyennes générales. 
Ces résultats montrent que les paysans ont une très bonne connaissance des stades physiologiques du riz qui sont sensibles aux attaques des foreurs de tiges.

\section{Connaissance des agriculteurs sur le niveau des pertes}

Sur la base des réponses recueillies lors de l'interview, $47 \%$ des paysans pensent que les pertes engendrées par les foreurs de tiges sont faibles $(<10 \%$ de la production), 38\% estiment qu'elles se situent entre $10-20 \%$ de la production, et seulement $15 \%$ trouvent qu'elles sont élevées (> $20 \%$ des récoltes). Au champ et précisément au moment de l'observation, $53 \%$ des producteurs ont estimé les pertes comme étant faibles, $29 \%$ les ont estimées comme moyennement grandes (10-20\%) et $18 \%$ les ont évalués comme importantes (tableau 3).

Au même moment, les notations faites par les agents enquêteurs dans les champs ont prouvé que les pertes dues aux attaques des foreurs de tige ne dépassaient pas $10 \%$. Ces résultats montrent que beaucoup de producteurs savent évaluer les pertes, mais que certains ont tendance à les surestimer.

\section{Discussion}

\section{Connaissance des agriculteurs sur les foreurs de tige du riz}

L'analyse des résultats présentés permet de situer clairement le niveau de connaissance des paysans sur les différents foreurs de tige du riz au Bénin.
La mouche à pédoncule, Diopsis spp., a été le foreur le plus reconnu sur l'ensemble des zones de production, mais en majorité par les agriculteurs des bas-fonds et ceux des zones irriguées. La fréquence et l'abondance de ces mouches dans les rizières, et aussi leur curieuse forme avec leurs yeux au bout des antennes seraient à la base de leur bonne identification par les paysans. Selon Cocherau (1978), les adultes des Diopsides sont fréquents pendant le jour et leur pullulation au champ se remarque facilement. En outre, de nombreuses recherches ont rapporté que ces mouches peuvent se rencontrer dans toutes les zones de culture du riz (Brenière, 1983), mais qu'elles sont plus abondantes dans les rizières irriguées (Heinrichs et al., 2004) et celles des bas-fonds (Appert et al., 1988 ; Olalekan, 2002). Le foreur rayé, C. zacconius, a été bien reconnu surtout dans les zones de plateau et dans les zones irriguées. La connaissance des paysans des plateaux pourrait s'expliquer par l'abondance de $C$. zacconius dans les rizières en culture pluviale, comme rapporté par Alam (1992), tandis que celle des paysans des zones irriguées serait le résultat de leur longue expérience en riziculture au Bénin.

Quant au foreur rose africain, S. calamistis, il a été reconnu par la majorité des producteurs en général, et en particulier par ceux des zones écologiques de plateau et de bas-fonds. Ce savoir paysan se justifie dans la mesure où le foreur rose serait prédominant aussi bien sur les plateaux (Alam, 1992) que dans les bas-fonds (Nwilene et al., 2008).

Le foreur blanc, $M$. Separatella, déclaré connu par $66 \%$ des producteurs lors de l'interview, ne l'a été que par seulement $47 \%$ au cours de la visite de terrain. Ce contraste serait dû au fait que les pro- ducteurs sont moins habitués à identifier les insectes sur collections et photos qu'au champ et auraient confondu les chenilles du foreur blanc avec celles d'autres espèces pendant l'interview.

Quant au foreur E. saccharina, il n'a pas été rencontré sur le terrain, mais l'hypothèse selon laquelle il attaquerait le riz dans les localités où cette culture se trouve dans les mêmes environnements que le maïs est soutenue par les travaux d'Appert (1988) et de Tran (1981). Ainsi, tout porte à croire que les producteurs le connaissent. Concernant enfin O. oryzivora, aucune investigation au champ ni aucune donnée de recherche antérieure n'ont signalé sa présence au Bénin. Il est normal que cette espèce ne soit pas reconnue par les agriculteurs.

Les enquêtes effectuées prouvent que les producteurs des départements du Zou, des Collines et du Couffo ont une bonne connaissance de S. calamistis, C. zacconius, Diopsis spp. et $M$. separatella. Selon plusieurs auteurs, ces espèces sont celles qui causent l'essentiel des dégâts sur le riz en Afrique de l'Ouest (Akinsola, 1975; Brenière, 1976 ; Hari et al., 1997 ; Tran, 1981 ; Alam, 1988; Diarisso, 1992 ; Heinrichs, 1998).

En résumé, il apparaît que les agriculteurs des principales zones rizicoles au Bénin de par leur savoir sur les foreurs de tiges seraient en mesure de participer aux opérations de prospection et de lutte contre eux.

\section{Connaissance des agriculteurs sur les dégâts et pertes dus aux foreurs de tige du riz}

Lors des interviews, les paysans avaient déclaré connaitre tous les symptômes

\section{Tableau 3. Connaissance des agriculteurs des niveaux de pertes induites par les foreurs de tige.}

Table 3. Farmer knowledge of loss levels due to stem borer damage.

\begin{tabular}{|c|c|c|c|c|c|c|c|c|}
\hline \multirow[b]{3}{*}{ Niveaux des dégâts } & \multicolumn{8}{|c|}{ Connaissance paysanne des niveaux de perte (\%) } \\
\hline & \multicolumn{2}{|c|}{ Bas-fonds } & \multicolumn{2}{|c|}{ Plateau } & \multicolumn{2}{|c|}{ Zone irriguée } & \multicolumn{2}{|c|}{ MG } \\
\hline & $\mathbf{R} \mathbf{I}$ & RC & $\mathbf{R} \mathbf{I}$ & RC & $\mathbf{R} \mathbf{I}$ & $\mathbf{R C}$ & $\mathbf{R} \mathbf{I}$ & $\mathbf{R C}$ \\
\hline Pertes faibles (< $10 \%)$ & 49 & 45 & 50 & 65 & 42 & 48 & 47 & 53 \\
\hline Pertes intermédiaires (10-20 \%) & 32 & 32 & 33 & 23 & 48 & 32 & 38 & 29 \\
\hline Pertes élevées (> 20 \%) & 19 & 23 & 17 & 12 & 10 & 20 & 15 & 18 \\
\hline Total & 100 & 100 & 100 & 100 & 100 & 100 & 100 & 100 \\
\hline
\end{tabular}

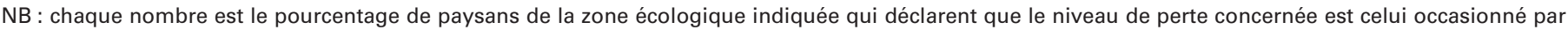
les foreurs.

$\mathrm{RI}$ : résultats suite aux interviews ; $\mathrm{RC}$ : résultats au champ ; $M G$ = moyennes générales. 
des dégâts des foreurs de tige, mais les observations au champ ont montré que les cours morts et les panicules blanches sont les seuls qui soient bien connus. En effet, ces deux types de symptômes sont facilement visibles. Le premier se traduit par un dessèchement de la feuille centrale (Cochereau, 1978) et le second par le dessèchement suivi d'une coloration blanche caractéristique des inflorescences (Heinrich et al., 2004). Quant aux symptômes de tunnels et de galles, ils ont été très peu ou pas identifiés.

En outre, les paysans ont déclaré que les stades tallage et épiaison étaient les plus vulnérables aux attaques des foreurs de tige et que les dégâts de cours morts et de panicules blanches étaient les plus importants économiquement. Ces résultats, confirmés par les observations au champ, reflètent la réalité d'autant plus que les coeurs morts apparaissent au stade tallage du riz et les panicules blanches au cours de l'épiaison (Descamps, 1957 ; Cochereau, 1978 ; Brenière, 1983 ; Heinrich et al., 2004), comme le disent les agriculteurs. Concernant l'estimation des pertes dues aux foreurs, il n'y avait pas de grande différence entre les résultats des interviews et ceux résultant de l'observation au champ, les estimations paysannes ayant été conformes aux résultats des notations effectuées par les enquêteurs. Ces résultats sont par ailleurs du même ordre de grandeur que les résultats obtenus dans des zones écologiques similaires d'autres régions d'Afrique où les pertes occasionnées par les foreurs ont été estimées entre 2 et $38 \%$ (Dakouo et al., 1992 ; Heinrichs, 1998).

Ces analyses prouvent que les producteurs des départements du Zou, des Collines et du Couffo ont une bonne connaissance des principaux dégâts des foreurs de tige du riz et seraient capables de participer à une opération d'investigation visant à les évaluer au champ.

\section{Conclusion}

L'étude a permis de démontrer qu'une bonne proportion des paysans des zones rizicoles du Bénin connaît les principales espèces de foreurs de tiges et leurs dégâts sur le riz. Dans quelques rares cas, une certaine confusion a été notée dans la relation entre type d'insecte et nature des dégâts, d'où un besoin de renforcement de capacités dans ce domaine. Dans l'ensemble, les résultats obtenus sont conformes à la réalité du terrain et sont soutenus par des recherches antérieures. Ces résultats devront permettre aux services d'encadrement agricole du pays et aux institutions de recherche de mieux impliquer les paysans dans les opérations de surveillance régulière et d'alerte précoce des foreurs de tige ainsi que dans les recherches participatives pour une lutte efficace contre ces ravageurs.

\section{Références}

Akinsola EA. Present status of different rice stem borers in plants of Nigeria. Rice Entomololy Newsletter $1975: 3$ p.

Alam MS. Seasonal abundance of rice stem borer species in upland rice and irrigated rice in Nigeria. Insect Sci Appl $1988 ; 9: 191-5$.

Alam MS. A survey of rice pests in Nigeria. Trop Pest Manage 1992 ; 38 : 115-8.

Appert J, Deuse, J. Le technicien d'agriculture tropical: Insectes nuisibles aux cultures vivrières et maraîchères. $2^{\mathrm{e}}$ éd. Paris: Ed G.-P. Maisonneuve et Larose; A.C.C.T, 1988.

Assigbe P. Développement participatif de technologies pour la gestion intégrée de la fertilité des sols rizicoles du centre Bénin. Actes de la seconde revue régionale de la recherche rizicole (4Rs 2002). Monrovia (Liberia) : ADRAO, 2003.

Brenière J. Reconnaissance des principaux lépidoptères du riz de I'Afrique de I'Ouest. Agronomie Tropicale 1976 ; 31 : 213-31.

Brenière J. Principaux ennemis du riz en Afrique de l'Ouest et leur contrôle. $2^{\mathrm{e}}$ éd. Monrovia (Liberia): ADRAO, 1983.

Chaudhary RC, Nanda JS, Tran DV. Guide d'identification des contraintes de terrain à la production de riz. Rome: FAO, 2003.
Cochereau P. Fluctuations des populations imaginales de Diopsis thoracica Westwood et de Diopsis apicalis Wstwood (Diptera, Diopsidea) en liaison avec la phénologie d'un riz de basfond à Bouaké (Côte d'Ivoire). Cahiers Orstom. Ser Biologie 1978 ; XIII : 45-58.

Dakouo D, Nacro S. Lutte contre les insectes ravageurs du riz au Burkina-Faso: bilan et perspectives, Vol. 1. Communication présentée au Deuxième séminaire sur la lutte intégrée contre les ennemis des cultures vivrières dans le Sahel. Bamako (Mali) : Ed Institut du Sahel, 1992.

Descamps M. Contribution a l'étude des diptères Diopsidae nuisibles au riz dans le nord Cameroun. Res. J Agric \& Biol Sci 1957 ; 4 : 83-93.

Diarisso NY. Les foreurs de tige du riz au Mali, Vol. 1. Communication présentée au Deuxième séminaire sur la lutte intégrée contre les ennemis des cultures vivrières dans le Sahel. Bamako (Mali) : Ed Institut du Sahel, 1992.

Friedberg C. Les savoirs populaires sur la nature. Sciences Humaines 1999; 24: Hors série: La dynamique des savoirs : 8-11.

Hari KP. Systèmes améliorés de riziculture pluviale. Archives de documents. Rome: FAO 1997. (www.fao.org/docrep/003/T0751F/ T0751F00.HTM)

Heinrichs EA. African Cereal Stem Borers : economic importance, taxonomy, natural enemies and control. London: Ed Polaszek ; CTA, 1998.

Heinrichs EA, Alberto T. Rice-Feeding Insects and Selected Natural Enemies in West Africa Biology, ecology, identification. Los Baños (Philippines) ; Abidjan (Côte d'Ivoire) : éditions IRRI/WARDA, 2004.

Nwilene FE, Jones MP, Brar DS, et al. Integrated pest management (IPM) strategies for NERICA varieties. In: The New Rice for Africa - a Compendium. Manuel. Cotonou : sn, 2008.

Olalekan OB. Management of major Insects Pests of Rice in Tanzania. Plant Protect Sci 2002 ; 38 : 108-13.

SAS Institute. SAS systems for Windows. Ver sion 9.1 Edition 2002-2003. Cary (NC, ÉtatsUnis) : SAS Institute Inc, 2003.

Tran M. Reconnaissance des principaux foreurs des tiges du riz, du maïs et de la canne à sucre en Côte d'Ivoire. Travaux et documentations de I'Orstom, $n^{\circ}$ 48. Paris: Orstom éditions, 1981

Umeh EDN, Joshi RC. Aspects of the biology ecology and natural biological control of the African rice gall midge, Orseolia oryzivora Harris and Gagné (Dipt.,Cecidomyiidae) in south east Nigeria. J Appl Entomol 1993 ; 116 : 391-8.

Velay F, Baudoin JP, Mergeai G. Caractérisation du savoir paysan sur les insectes nuisibles du pois d'Angole (Cajanus cajan (L.) Millsp.) dans le Nord de I'Ouganda. Biotechnol Agron Soc Environ 2001; 5 : 105-14. 